\title{
Gut microbial metabolites in obesity, NAFLD and T2DM
}

Citation for published version (APA):

Canfora, E. E., Meex, R. C. R., Venema, K., \& Blaak, E. E. (2019). Gut microbial metabolites in obesity, NAFLD and T2DM. Nature Reviews Endocrinology, 15(5), 261-273. https://doi.org/10.1038/s41574-0190156-z

Document status and date:

Published: 01/05/2019

DOI:

10.1038/s41574-019-0156-z

Document Version:

Publisher's PDF, also known as Version of record

Document license:

Taverne

Please check the document version of this publication:

- A submitted manuscript is the version of the article upon submission and before peer-review. There can be important differences between the submitted version and the official published version of record.

People interested in the research are advised to contact the author for the final version of the publication, or visit the DOI to the publisher's website.

- The final author version and the galley proof are versions of the publication after peer review.

- The final published version features the final layout of the paper including the volume, issue and page numbers.

Link to publication

\footnotetext{
General rights rights.

- You may freely distribute the URL identifying the publication in the public portal. please follow below link for the End User Agreement:

www.umlib.nl/taverne-license

Take down policy

If you believe that this document breaches copyright please contact us at:

repository@maastrichtuniversity.nl

providing details and we will investigate your claim.
}

Copyright and moral rights for the publications made accessible in the public portal are retained by the authors and/or other copyright owners and it is a condition of accessing publications that users recognise and abide by the legal requirements associated with these

- Users may download and print one copy of any publication from the public portal for the purpose of private study or research.

- You may not further distribute the material or use it for any profit-making activity or commercial gain

If the publication is distributed under the terms of Article $25 \mathrm{fa}$ of the Dutch Copyright Act, indicated by the "Taverne" license above, 


\section{REVIEWS}

\section{Gut microbial metabolites in obesity, NAFLD and T2DM}

Emanuel E. Canfora ${ }^{0}$, Ruth C. R. Meex, Koen Venema and Ellen E. Blaak ${ }^{*}$ *

Abstract | Evidence is accumulating that the gut microbiome is involved in the aetiology of obesity and obesity-related complications such as nonalcoholic fatty liver disease (NAFLD), insulin resistance and type 2 diabetes mellitus (T2DM). The gut microbiota is able to ferment indigestible carbohydrates (for example, dietary fibre), thereby yielding important metabolites such as short-chain fatty acids and succinate. Numerous animal studies and a handful of human studies suggest a beneficial role of these metabolites in the prevention and treatment of obesity and its comorbidities. Interestingly, the more distal colonic microbiota primarily ferments peptides and proteins, as availability of fermentable fibre, the major energy source for the microbiota, is limited here. This proteolytic fermentation yields mainly harmful products such as ammonia, phenols and branched-chain fatty acids, which might be detrimental for host gut and metabolic health. Therefore, a switch from proteolytic to saccharolytic fermentation could be of major interest for the prevention and/or treatment of metabolic diseases. This Review focuses on the role of products derived from microbial carbohydrate and protein fermentation in relation to obesity and obesity-associated insulin resistance, T2DM and NAFLD, and discusses the mechanisms involved.

The worldwide prevalence of obesity, obesity-associated nonalcoholic fatty liver disease (NAFLD), insulin resistance and type 2 diabetes mellitus (T2DM) has grown dramatically since the $1980 \mathrm{~s}^{1-4}$. Driven by easy access to energy-dense foods and increasingly sedentary lifestyles, obesity is a major global health and socio-economic problem in the 21 st century ${ }^{1,5}$.

Obesity develops in the setting of a chronic positive energy balance (where energy intake exceeds energy expenditure). In this situation, the adipose tissue exceeds its buffering capacity to store all surplus energy as triglycerides, resulting in lipid overflow into the circulation. This increased supply of lipids to nonadipose tissues such as the liver and skeletal muscle, together with an impaired ability to adjust lipid oxidation to lipid supply ('metabolic inflexibility ${ }^{6}$ ), results in ectopic fat storage ${ }^{7}$. Together with the reduced adipose tissue lipid-buffering capacity, adipose tissue inflammation develops, resulting in increased production and secretion of pro-inflammatory adipokines ${ }^{8}$. Altogether, these obesity-related disturbances in tissue and organ function and crosstalk contribute to the development of peripheral and hepatic insulin resistance and the development of T2DM and NAFLD ${ }^{7,9}$.

Over the past 15 years, the gut microbiome has emerged as an important regulator of host energy metabolism and substrate metabolism ${ }^{10-13}$. Abnormalities in gut microbiota composition and/or its functionality might contribute to a disturbed energy and substrate metabolism, including effects on metabolism in adipose tissue, muscle and liver. In addition, the gut microbiota has been associated with the development of obesity-related chronic low-grade inflammation ${ }^{14-16}$. A putative nutritional strategy to modulate the gut microbiome, thereby preventing and alleviating these metabolic disorders, is to enhance the availability of dietary fibre to colonic microorganisms $s^{17-21}$. The fermentation of these indigestible carbohydrates (saccharolytic fermentation) yields the short-chain fatty acids (SCFAs) acetate, propionate and butyrate as end products, as well as other products such as succinate ${ }^{22,23}$. Animal studies suggest that SCFAs and succinate have an important role in the prevention and treatment of obesity-associated insulin resistance ${ }^{1924-29}$. Furthermore, human evidence for a beneficial effect of SCFAs on body weight control, inflammatory status and insulin sensitivity, as well as in glucose and lipid homeostasis, is increasing ${ }^{17,30-35}$.

Interestingly, the distal colonic microbiota uses mainly residual peptides and proteins to gain energy (proteolytic fermentation) ${ }^{36}$ because its preferred fuel, fermentable carbohydrates, is already utilized in the more proximal colon ${ }^{36}$. Of note, microbial fermentation of proteins yields a great diversity of metabolites, which are most often considered detrimental for gut integrity and metabolic health ${ }^{17,37}$. 


\section{Key points \\ - Gut microbial metabolites such as short-chain fatty acids (SCFAs) and succinate, which are derived from the fermentation of dietary fibre, have important metabolic functions. \\ - SCFAs and succinate might prevent obesity by increasing energy expenditure, increasing anorexic hormone production and improving appetite regulation. \\ - SCFAs have a crucial role in gut homeostasis, adipose tissue and liver substrate metabolism and function, through which they can prevent the progression of type 2 diabetes mellitus (T2DM) and nonalcoholic fatty liver disease (NAFLD). \\ - The site of microbial SCFA production in the colon might be an important determinant for the aforementioned beneficial effects. \\ - The microbial metabolites derived from protein fermentation, which are mainly produced in the distal colon, are most often considered detrimental for gut integrity and metabolic health. \\ - Providing mixtures of dietary fibres to increase distal colonic microbial carbohydrate fermentation and thereby inhibit protein fermentation might be a putative target to ameliorate obesity, T2DM and NAFLD.}

In this Review, we focus on the role of gut microbial metabolites derived from carbohydrate fermentation (including acetate, propionate, butyrate, succinate and ethanol) and protein fermentation (including ammonia, phenolic compounds, indoles, hydrogen sulfide and branched-chain fatty acids (BCFAs)) in body weight control, NAFLD, insulin resistance and T2DM. Further microbial metabolites, such as dimethylamine and trimethylamine, lipopolysaccharide and secondary bile acids, which are strongly involved in the gut hostmicroorganism metabolic axis and the development of metabolic disorders, have been excellently reviewed elsewhere $^{38-40}$.

We review human studies that provide evidence for a role of microbial metabolites in these metabolic disturbances and diseases and we discuss the putative mechanisms involved. We aim to identify gaps in the literature and to provide leads for future research in the emerging field of the gut microbiome in relation to the development of human metabolic diseases.

\section{Microbial fermentation in the colon}

The human gut microbiome contains 500-1,000 bacterial species with an estimated 2,000,000 genes, which outnumbers the number of human genes by $\sim 100$ times $^{41}$. The small intestine is colonized with a low abundance of mainly facultative anaerobic microorganisms (microbial density $<10^{7}$ microorganisms per gram of content). By contrast, the caecum and colon are inhabited by up to $10^{12}$ microorganisms per gram of content ${ }^{41}$. The microbial composition and abundance are dependent on a number of intrinsic factors (for example, $\mathrm{pH}$, gut motility, mucus and antimicrobial peptides) and extrinsic factors such as medications (for example, antibiotics, laxatives, opioids and NSAIDs) and dietary components $^{42-44}$.

Indigestible foods have a major role in shaping the composition of the gut microbiota. Fermentable carbohydrates, including malabsorbed carbohydrates, resistant starch and prebiotics, are preferred substrates for most members of the colonic microbiota as they like to incorporate available proteins and amino acids into their own biomass and enzyme machinery. However, as they travel from the proximal portion of the colon to the distal portion, fermentable carbohydrates become depleted (especially with current Western diets that contain low amounts of indigestible carbohydrates) and microorganisms switch to protein fermentation.

Not much is known about the communities and microbiological networks that produce saccharolytic and proteolytic metabolites. Certain members of the microbiota have been shown to contribute to particular microbial pathways (FIG. 1). However, because most members of the microbiota are difficult to culture and hence are challenging to investigate in detail, knowledge of microbial pathways is lacking for most of the $\sim 250$ members that make up an individual's microbiota, and we have little understanding of the redundancy that exists between different microorganisms. Moreover, individuals share only a limited number of microorganisms: 57 microorganisms have been shown to constitute the core species, being present in $\geq 90 \%$ of the individuals tested ${ }^{45}$. Therefore, microbial communities and microbiological networks might differ between individuals, depending on the degree of redundancy present within the gut microbiota.

The fermentation of dietary fibre yields high amounts of SCFAs, as well as lactate, succinate and gases such as methane, carbon dioxide and hydrogen ${ }^{46}$. As indicated above, most saccharolytic fermentation occurs in the proximal colon and is mostly finalized in the transverse colon. Hence, only a minor amount of fermentable fibre reaches the distal colonic site. This pattern is also reflected in the concentrations of saccharolytic metabolites found in sudden-death victims, in which a progressive decline in SCFA, lactate and succinate concentrations was shown in passing down the colon ${ }^{47}$.

The distal colonic microbiota is therefore specialized towards gaining energy from the fermentation of residual peptides and proteins. Proteolytic fermentation also yields SCFAs. However, the contribution of protein fermentation to the amount of SCFAs produced in the distal colon is not known and would require experiments using stable isotope-labelled proteins, for example. Furthermore, in comparison to the fermentation of carbohydrates, proteolytic fermentation yields a more diverse range of metabolites, including the following: gaseous products such as hydrogen, methane, carbon dioxide and hydrogen sulfide; the BCFAs isobutyrate, 2-methylbutyrate and isovalerate derived from fermentation of branched-chain amino acids (BCAAs); and phenolic and indolic compounds derived from microbial fermentation of aromatic amino acids (AAAs) ${ }^{37}$. Many of these compounds are toxic and have been considered as being detrimental for colonic and metabolic health ${ }^{17,37,48}$. However, metabolites such as indole and hydrogen sulfide might beneficially affect gut and peripheral tissue function ${ }^{49,50}$. In addition, microbial proteolysis can yield BCAAs and AAAs, which can be further metabolized by microbial cross-feeding (nutritional interdependence of microbial species $)^{44,48}$. Disturbances in these cross-feeding pathways lead to increased absorption of these amino acids and are associated with impairments in gut integrity and insulin resistance ${ }^{44,48,51}$. 


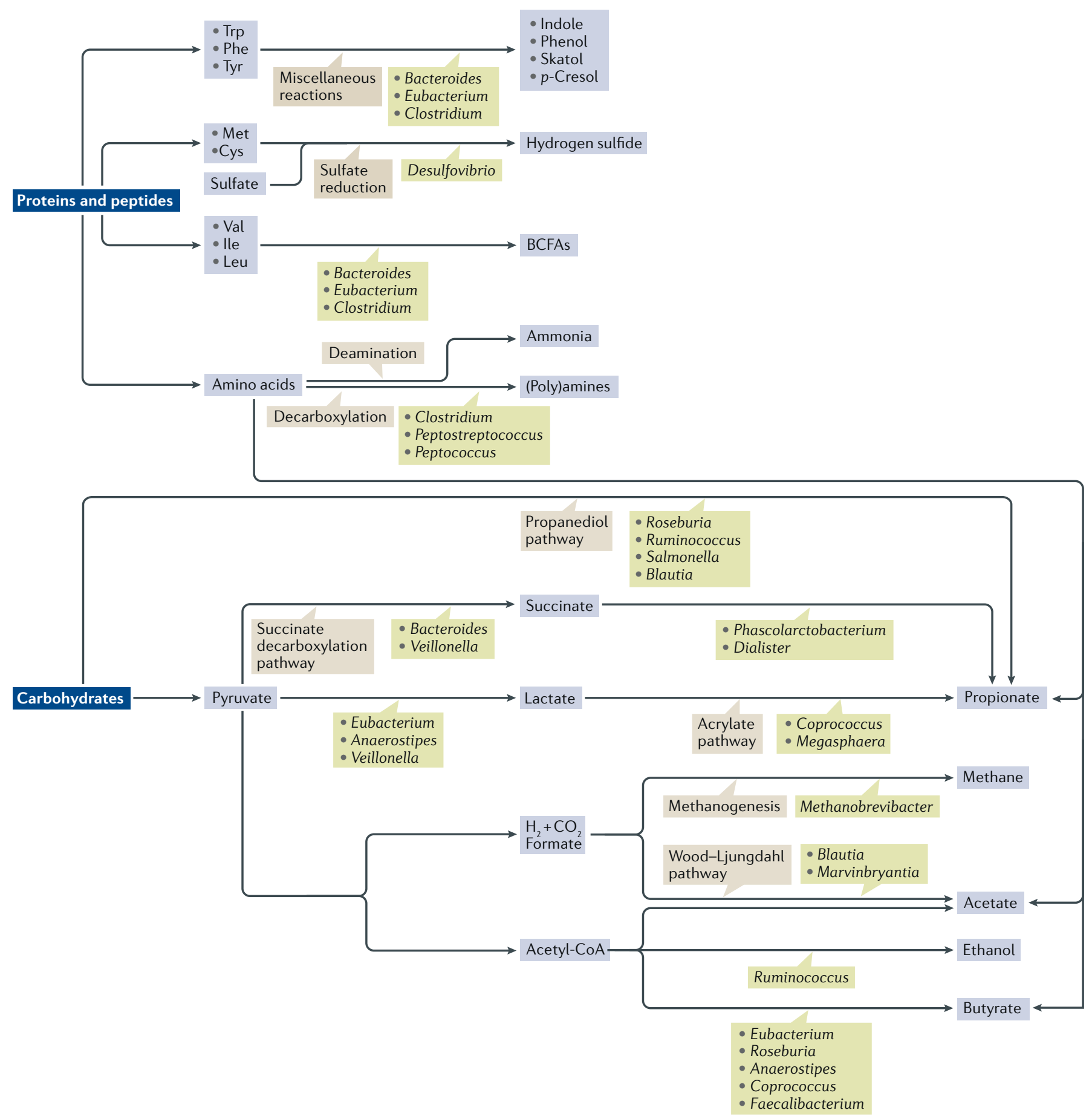

Fig. 1 | Microbial communities and microbiological networks involved in saccharolytic and proteolytic metabolite production. The figure shows an overview of the functional groups of gut microorganisms, major metabolic pathways and intermediates involved in the production of metabolites derived from the fermentation of carbohydrates and proteins, including the saccharolytic products short-chain fatty acids, succinate and ethanol as well as proteolytic metabolites including ammonia, indolic and phenolic compounds, hydrogen sulfide, amines and branched-chain fatty acids (BCFAs).

\section{Colonic metabolite concentrations}

In individuals who died suddenly, total SCFA concentrations (mean \pm s.e.) of $123 \pm 12 \mathrm{mmol} / \mathrm{kg}$ luminal content in the proximal colon and $100 \pm 30 \mathrm{mmol} / \mathrm{kg}$ luminal content in the sigmoid and rectum region have been found with a molar ratio of acetate to propionate to butyrate of roughly $3: 1: 1\left(\right.$ REF. $\left.^{47}\right)$. These data already indicate that SCFA production rates are highly variable in the colon and strongly depend on nutritional status, amount of complex foods in the diet, gut microbial composition and colonic transit time $e^{52-54}$. In the study of SCFA concentrations in sudden-death victims, succinate concentrations were also measured in the colon ${ }^{47}$. Concentrations of succinate in the 
proximal and sigmoid colon were $3.1 \pm 0.9 \mathrm{mmol} / \mathrm{kg}$ and $2.1 \pm 1.0 \mathrm{mmol} / \mathrm{kg}$ luminal content, respectively ${ }^{47}$. Whereas colonic concentrations of SCFAs and succinate are found in concentrations of millimoles per kilogram luminal content, a completely different situation prevails in the blood compartment, where concentrations of SCFAs and succinate are in the micromole per litre range. Succinate concentration in the blood plasma is $\sim 5-200 \mu \mathrm{mol} / 1\left(\mathrm{REF}^{55}\right)$. Acetate is the most abundant SCFA systemically and its serum and plasma mean concentrations range from $5 \mu \mathrm{mol} / \mathrm{l}$ to $220 \mu \mathrm{mol} / \mathrm{l}\left(\mathrm{REFS}^{56,57}\right.$ ). Propionate and butyrate are found at much lower concentrations of $\leq 13 \mu \mathrm{mol} / \mathrm{l}$ and $\leq 12 \mu \mathrm{mol} / \mathrm{l}$, respectively $^{56,57}$. Circulating SCFA concentrations strongly depend on diet, gut microbial SCFA production, absorption, splanchnic extraction and hepatic metabolism. Therefore, human in vivo studies should use stable, isotopically labelled fermentable fibres and SCFAs $s^{58}$ in order to help to identify the actual contribution of microorganism-derived SCFAs.

Interestingly, the greatest release of SCFAs from the gut into the blood system has been demonstrated to occur in the distal colon ${ }^{59}$. SCFAs absorbed in the proximal colon are transported via the portal vein to the liver. Butyrate and propionate are highly extracted and metabolized by the liver, whereas acetate gets extracted in a lower percentage by the liver and therefore reaches the systemic circulation in significantly higher amounts ${ }^{47,57}$.

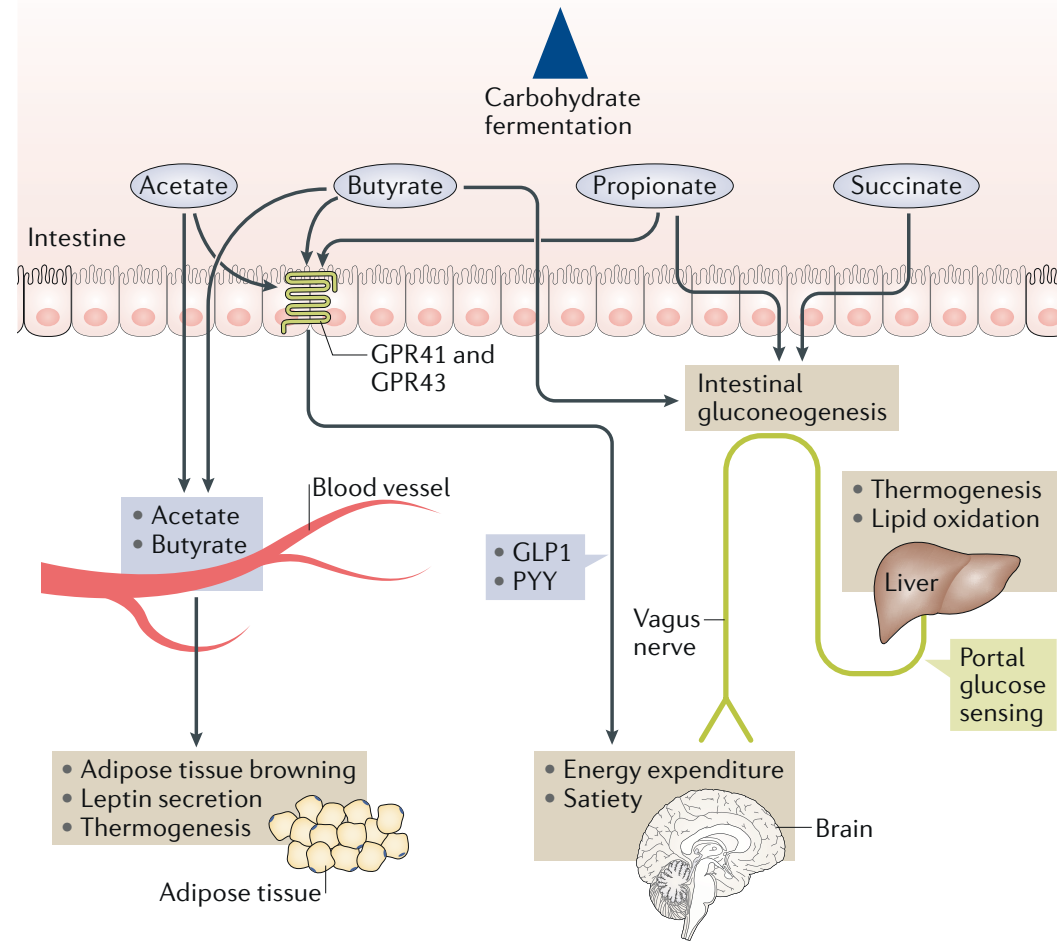

Fig. 2 | Metabolites derived from carbohydrate fermentation in relation to body weight control. Propionate, butyrate and succinate induce intestinal gluconeogenesis, thereby beneficially regulating energy homeostasis. Acetate and butyrate have also been demonstrated to induce satiety via a central mechanism and to increase thermogenesis in adipose tissue and liver as well as to induce adipose tissue browning and leptin secretion. In addition, acetate, propionate and butyrate stimulate the secretion of the satiety hormones glucagon-like peptide 1 (GLP1) and peptide YY (PYY) in a $\mathrm{G}$ protein-coupled receptor (GPR)-dependent manner.
By contrast, SCFAs absorbed in the distal colon can bypass the liver via the pelvic plexus, which drains into the vena cava inferior, thereby reaching the systemic circulation directly. This finding suggests that a higher amount of microbial metabolites will reach peripheral organs if administered or produced in the distal colon.

Concentrations of proteolytic metabolites have not been greatly studied and have mainly been determined in faecal samples. In healthy individuals, mean concentrations of total BCFAs, ammonia, p-cresol, total phenols (phenol and 4-ethylphenol), total indoles (indole, 2-methylindole, 3-methylindole (skatole) and 2,3-dimethylindole), total amines (agmatine, cadaverine, histamine, phenylethylamine, putrescine, spermidine, spermine, tryptamine and tyramine) and dimethyl disulfide were $18.87 \mathrm{mmol} / \mathrm{kg}$ dry matter, $160.93 \mathrm{mmol} / \mathrm{kg}$ dry matter, $2.12 \mathrm{mmol} / \mathrm{kg}$ dry matter, $2.39 \mathrm{mmol} / \mathrm{kg}$ dry matter, $1.56 \mathrm{mmol} / \mathrm{kg}$ dry matter, $22.32 \mathrm{mmol} / \mathrm{kg}$ dry matter and $1.97 \mathrm{mmol} / \mathrm{kg}$ dry matter, respectively ${ }^{60}$. Studies in pigs $^{61}$ and humans $\mathrm{s}^{37,62}$ demonstrated that increased availability of dietary proteins and diminished availability of fermentable fibres increased the production of metabolites derived from proteolytic fermentation in the colon. Furthermore, in 1992, seminal data from two individuals who died suddenly showed that concentrations of soluble proteins and proteolytic compounds including ammonia, $p$-cresol, phenylacetate and phenylpropionate are significantly lower in the proximal colon than in the sigmoid and rectum regions ${ }^{63}$.

\section{Microbial metabolites in weight control}

This section focuses on the impact of the saccharolytic fermentation products (SCFAs and succinate) on body weight control, including effects on appetite regulation and energy intake, energy expenditure and lipid oxidation. Literature on a direct role of metabolites derived from protein fermentation on body weight control is thus far lacking. A schematic view on the role of SCFAs and succinate is presented (FIG. 2).

SCFAs in appetite regulation and energy intake. SCFAs affect appetite and energy intake via various mechanisms. One of the best studied mechanisms is the ability of SCFAs to stimulate the production of satiety hormones. In vitro studies showed that SCFAs stimulate the secretion of peptide YY (PYY) and glucagon-like peptide 1 (GLP1) from enteroendocrine cells through the $G$ protein-coupled receptors (GPRs) GPR41 and GPR43 (also known as FFAR3 and FFAR2, respectively) in rodent ${ }^{64,65}$ and human ${ }^{66,67}$ cell lines. Furthermore, results of rodent in vivo studies confirmed that SCFAs stimulate the release of GLP1 and PYY $^{64,68,69}$. In addition, SCFAs can stimulate the secretion of the adipose-tissue-derived satiety hormone leptin, as demonstrated in mouse ${ }^{70}$, bovine ${ }^{71}$ and human adipocytes $^{72}$ in vitro. Moreover, human in vivo studies showed that acute rectal infusions of sodium acetate ${ }^{34,73}$ and SCFA mixtures ${ }^{30}$ increased circulating concentrations of PYY in individuals who were overweight. In line with these findings, an interesting study in patients with obesity showed that acute oral intake of $10 \mathrm{~g}$ of inulin propionate ester increased postprandial plasma 
concentrations of PYY and GLP1, which resulted in a $14 \%$ reduction in food intake (versus $10 \mathrm{~g}$ inulin alone $^{32}$. However, despite the positive effects on body weight control, no differences in satiety hormones were seen after 24 weeks of inulin propionate ester supplementation at $10 \mathrm{~g}$ per day when compared with $10 \mathrm{~g}$ per day inulin alone ${ }^{32}$. Therefore, although the metabolic effects of (targeted) SCFA delivery or production seem promising, the long-term metabolic consequences, in particular the effects on satiety-stimulating hormones, require further study.

Elegant rodent studies demonstrated that SCFAs also have a suppressive effect on appetite and energy intake via central nervous system-related mechanisms and the gut-brain axis ${ }^{19,25,29,74}$. A study in mice demonstrated that colonically derived ${ }^{11} \mathrm{C}$-acetate can pass through the blood-brain barrier and reach the hypothalamus ${ }^{25}$. Increased hypothalamic acetate availability induced a glutamate-glutamine transcellular cycle and increased the production of lactate and GABA, which resulted in the suppression of appetite and energy intake ${ }^{25}$. In line with this finding, a 2017 study in mice indicated that chronic oral butyrate administration prevented diet-induced obesity, progression of NAFLD and insulin resistance ${ }^{29}$. These effects were mainly related to reduced food intake, via a butyrate-induced suppression of the activity of orexigenic neurons that express neuropeptide $\mathrm{Y}$ in the hypothalamus, and diminished neuronal activity within the nucleus tractus solitarius and dorsal vagal complex in the brainstem ${ }^{29}$.

Intraperitoneal injection of acetate, propionate and butyrate has been shown to inhibit energy intake in mice via a mechanism related to vagal afferent stimulation $^{74}$. Indeed, vagal afferent chemoreceptors might have a central role in SCFA-induced appetite regulation by sensing SCFAs or gut hormones such as PYY and GLP1. The importance of the vagus nerve in GLP1related energy intake regulation was demonstrated by a study showing that the ability of GLP1 to reduce food intake was completely lost in humans who had undergone truncal vagotomy ${ }^{75}$. However, the relevance of a direct SCFA-induced vagal afferent neuron stimulation in human energy homeostasis warrants further investigation. Furthermore, a series of elegant experiments in mice demonstrated that propionate and butyrate prevented obesity and insulin resistance via the induction of intestinal gluconeogenesis $(\mathrm{IGN})^{19}$. Whereas butyrate directly induced IGN in enterocytes, propionate activated GPR41 in the periportal afferent neural system to induce IGN. The increased IGN resulted in decreased hepatic glucose production and improved energy homeostasis ${ }^{19}$. Whether IGN has a central role in metabolic health in humans still needs to be determined.

A first indication that SCFAs might affect central appetite regulation in humans is shown by an acute study in healthy individuals ${ }^{76}$. Using functional MRI, this study demonstrated that colonic propionate delivery (using $10 \mathrm{~g}$ inulin propionate ester) diminished activity in the caudate and the nucleus accumbens (both brain regions that are linked to food cravings) when the participants looked at high-calorie foods. These central effects were directly related to decreased ad libitum food intake $^{76}$. Whether this finding is true in the long term and whether it can be translated to a more metabolically disturbed phenotype should be investigated in the future via the use of isotopically labelled SCFAs and advanced imaging techniques ${ }^{77}$.

SCFAs affect energy expenditure. SCFAs might also beneficially affect body weight by influencing energy expenditure. In obese mice, oral administration of butyrate resulted in decreases in body weight, mainly driven by an increase in energy expenditure and lipid oxidation $^{78}$. This effect was associated with the upregulation of expression of the thermogenesis-related genes peroxisome proliferator-activated receptor- $\gamma$ (PPAR $\gamma)$ co-activator $1 \alpha$ (PPARGC1A, encoding PGC1 $\alpha)$ and uncoupling protein 1 ( $U C P 1)$ in brown adipose tis$\operatorname{sue}^{78}$. In line with this finding, nanoparticle-delivered acetate to white adipose tissue induced adipose tissue browning and increased thermogenic capacity, thereby preventing adiposity in mice ${ }^{79}$. In addition, intragastric and oral SCFA administration to mice fed a highfat diet decreased total body fat content and hepatic fat accumulation without changing food intake ${ }^{24,80}$. This finding has been linked with increased expression of the thermogenesis-related proteins acetyl-CoA oxidase, carnitine palmitoyltransferase I and UCP2 in the liver and adipose tissue $\mathrm{e}^{24,80}$. These animal data provide important evidence for SCFA-induced upregulation of genes related to thermogenesis and lipid oxidation resulting in the prevention of weight gain and adiposity. However, the relevance and contribution of uncoupling processes and/or adipose tissue browning to energy homeostasis and control of body weight in humans is not yet fully understood. Interestingly, human in vivo data indicated that acute infusions of acetate as well as SCFA mixtures of acetate, propionate and butyrate in the distal part of the colon increased fasting lipid oxidation and resting energy expenditure in volunteers who were overweight or obese ${ }^{30,34}$. In addition, a 2018 in vivo study in healthy men showed that acute oral propionate administration raised resting energy expenditure and fasting lipid oxidation, independent of insulin and glucose levels and sympathetic nervous system activity ${ }^{33}$. Unfortunately, these acute studies did not provide mechanistic insight. In vivo, stable isotope tracers as well as analysis of tissue biopsy samples of adipose tissue and skeletal muscle could help to elaborate on the underlying mechanisms. In addition, mechanistic cell culture studies should be performed to further detect key regulators and pathways involved in human-derived adipocyte, hepatocyte or skeletal muscle cell models. Furthermore, additional human intervention studies are required to investigate whether these SCFAinduced improvements in oxidative metabolism translate into long-term benefits in weight control.

SCFAs and energy harvesting. Whereas the mechanisms described above suggest beneficial effects of SCFAs on the control of body weight and adiposity, data are available suggesting that microbial SCFA production and metabolism by the host lead to extra energy harvest from the diet. Human cross-sectional studies showed 
that individuals with obesity had higher faecal concentrations of SCFAs than individuals who were lean ${ }^{81,82}$. However, whether faecal concentrations are the best predictor of microbial SCFA production is debatable, as only $5-10 \%$ of the produced SCFAs are excreted. More direct evidence for a role of microbial-derived SCFAs in energy harvest comes from a study in genetically obese mice ${ }^{83}$. These mice had increased amounts of SCFAs in their caecum and reduced faecal energy content compared with their lean littermates ${ }^{83}$. However, rodent experiments with isotopically labelled SCFAs demonstrated that absorption of SCFAs, not caecal concentration of SCFAs, is relevant for metabolic health ${ }^{84}$. Interestingly, this study showed an inverse correlation between caecal SCFA absorption (as calculated via a mathematical model) and body weight and percentage body $\mathrm{fat}^{84}$. Thus far, no human data are available quantifying the contribution of microbial SCFAs to host energy balance, taking into account both the extra energy provided by SCFAs as well as the SCFA-induced increases in energy expenditure and decreases in food (and thus energy) intake.

Succinate in energy homeostasis. Succinate, classically described as being an intermediate in the microbial synthesis of propionate, has been shown to have an interesting role in control of body weight ${ }^{27}$. In mice, the caecal concentration of succinate was increased after fructooligosaccharide supplementation. Similar to the previously described mechanism for propionate, increased caecal levels of succinate resulted in the activation of IGN, which prevented the obese and glucose-intolerant phenotype of mice fed a high-fat and high-sucrose $\operatorname{diet}^{27}$. However, whether the beneficial effect of succinate can be translated into humans requires further investigation. Controversial data were reported in a 2018 explorative study in humans with a wide range of BMIs ${ }^{55}$. This study showed that an increased abundance of succinate-producing bacteria and a decreased abundance of succinate-consuming bacteria as well as increased circulating concentrations of succinate were all associated with obesity and impairments in glucose homeostasis. Interestingly, diet-induced weight loss reduced the abundance of microorganisms related to succinate metabolism and correlated with reduced circulating concentrations of succinate ${ }^{55}$. Whether succinate represents a microbial-derived disease-causing metabolite in humans, or whether the increased circulating concentrations of succinate are a consequence of obesity-related dysbiosis and impairments in gut permeability, remains to be determined.

Human intervention studies in body weight control. Human epidemiological and intervention studies demonstrated an inverse relationship between dietary fibre intake and body weight ${ }^{20,85-87}$. Indeed, several dietary intervention studies have demonstrated that long-term supplementation with fermentable fibres increases the production of satiety-stimulating hormones and reduces food intake in humans ${ }^{21,88-90}$.

However, owing to the complex and multiple interactions between dietary fibre and human metabolism, it is not clear to what extent these effects are related to saccharolytic microbial metabolites per se or to other fibre effects such as their effects on macronutrient absorption and glycaemic response, faecal bulking, changes in gastrointestinal transit time, direct effects on satiety hormones or interactions with the gut immune system.

Direct evidence indicating a beneficial role of SCFAs in body weight control is thus far limited. In a study in which faecal microbiota were transplanted from human twins discordant for obesity to germ-free mice, mice receiving the obese microbiota transplant showed increased adiposity, reduced caecal concentrations of SCFAs and increased monosaccharide and disaccharide concentrations after dietary fibre intake ${ }^{91}$. Furthermore, mice harbouring the transplanted obese microbiome exhibited higher expression of microbial genes involved in the biosynthesis of several amino acids and had higher serum levels of BCAAs than mice harbouring the lean microbiota ${ }^{91}$. This finding suggests that the microbiota from individuals with obesity has a lower capacity to completely ferment fermentable fibres and that microbial-derived SCFAs are of major importance for host energy homeostasis. In addition, these data indicate that the microbiome of individuals with obesity can contribute to metabolites (such as BCAAs) that are associated with the obese, insulin-resistant phenotype $^{92}$.

Furthermore, another study showed that 12 weeks of daily intake of oral apple cider vinegar containing $1.5 \mathrm{~g}$ acetate was associated with reduced body weight, reduced total body fat percentage and reduced visceral fat percentage in patients with obesity ${ }^{93}$. Finally, in adults with obesity, $10 \mathrm{~g}$ of daily inulin propionate ester intake for 24 weeks prevented body weight gain and reduced intra-abdominal adipose tissue compared with $10 \mathrm{~g}$ per day of inulin alone ${ }^{32}$. As described above, several animal and acute human studies also demonstrated beneficial effects of enhanced colonic acetate and butyrate availability on mechanisms related to body weight control. Thus, the interesting approach of esterifying propionate to a fermentable fibre ${ }^{32}$, thereby increasing the availability of SCFAs in the ileum and colon in a controlled manner, should also be tested with acetate and butyrate. Overall, more human studies are needed to demonstrate whether the SCFA-related benefits in appetite regulation and energy homeostasis translate into weight loss or prevention of weight gain in the long term.

\section{Microbial metabolites in NAFLD}

NAFLD is highly prevalent in individuals with obesityand is strongly associated with insulin resistance and T2DM ${ }^{94}$. NAFLD has become the most common liver disease in Western countries, and its incidence is still increasing ${ }^{2}$.

The gut and liver are intrinsically connected and strongly depend on each other in terms of metabolic functioning. Not surprisingly, disturbances in the gut-liver axis, including increased gut permeability and dysbiosis, are linked to NAFLD. Microbial products derived from saccharolytic and proteolytic fermentation might affect the gut-liver axis via multiple mechanisms, as described below, and hence contribute to NAFLD pathogenesis. 
SCFAs in NAFLD. Studies in rodents suggest that SCFAs have a beneficial role in hepatic metabolism and functioning. In particular, butyrate has a major role in maintaining gut integrity by upregulating the expression of tight junction proteins and mucins, which improves the gut barrier function and prevents the migration of toxic compounds, including ethanol and pro-inflammatory molecules, to the liver ${ }^{95,96}$. One of the best studied microorganism-derived pro-inflammatory components is lipopolysaccharide, which is continuously released into the colon upon the death of Gram-negative bacteria. Rodent ${ }^{14-16}$ and human association ${ }^{97-99}$ studies have demonstrated that microorganism-derived lipopolysaccharide has a key role in the progression of metabolic diseases such as NAFLD, insulin resistance and T2DM. Furthermore, butyrate has been demonstrated to suppress inflammation in mice via enhanced differentiation of colonic anti-inflammatory regulatory $\mathrm{T}$ cells ${ }^{100,101}$ and induced the NLRP3 inflammasome ${ }^{101}$ in a GPR-coupled manner, which have all been shown to be involved in NAFLD progression ${ }^{16,102}$.

More direct evidence for a beneficial function of SCFAs in the prevention of NAFLD was provided by a number of in vivo studies that used rodent mod$\mathrm{els}^{24,28,79,80,103,104}$. Supplementation of acetate, propionate or butyrate to animals reduced hepatic fat accumulation, decreased hepatic inflammation and suppressed cholesterol synthesis. The underlying mechanisms are related to increased hepatic lipid oxidation via an AMPK-acetyl-CoA carboxylase pathway, reduced tumour necrosis factor (TNF) expression, increased glycogen storage and reduced hepatic fatty acid synthase activity $^{24,28,79,80,103,104}$.

However, owing to the complexity and invasiveness of such a study, no human in vivo data on a direct link between microbial-derived SCFAs, liver function and NAFLD progression are available.

Microbially derived ethanol in NAFLD. Ethanol is a microbial metabolite derived from saccharolytic fermentation and microbial cross-feeding. Patients with nonalcoholic steatohepatitis (NASH) and obesity show an increased abundance of ethanol-producing bacteria in faeces as well as increased concentrations of ethanol in the systemic circulation and breath ${ }^{105-107}$. Gut-bacteriaderived ethanol (or the oxidized metabolite acetaldehyde) is possibly involved in NAFLD progression via direct toxic effects on hepatic cells ${ }^{108,109}$, via impairments in gut barrier function resulting in increased portal endotoxaemia ${ }^{110}$, and via the upregulation of nuclear factor- $\kappa B$ $(\mathrm{NF}-\kappa \mathrm{B})$ signalling pathways in peripheral cells ${ }^{111,112}$. Future research needs to demonstrate whether patients with NAFLD should avoid the consumption of specific indigestible carbohydrates and instead consume other carbohydrates that do not increase microbial ethanol production and reduce high ethanol-producing bacteria.

Proteolytic metabolites in NAFLD. Protein fermentative strains of the gut microbiota might be involved in mediating pro-inflammatory responses and NAFLD progression $^{113}$. In particular, hydrogen sulfide, ammonia and phenolic compounds have been associated with detrimental effects on gut epithelial health and gut permeability, as shown in rodent models fed with high-protein diets ${ }^{14,115}$. Therefore, these proteolytic metabolites might indirectly contribute to NAFLD progression via increased translocation of toxic compounds to the liver. An interesting study ${ }^{116}$ using a germ-free mouse model indicated a potential direct link between products of microbial protein fermentation and NAFLD. Mice fed a high-fat diet developed hepatic macrovesicular steatosis after colonization with microbiota of diabetic mice, whereas the control mice that were treated with microbiota of normoglycaemic mice developed only low-level steatosis. In comparison with control mice, mice with macrovesicular steatosis had markedly increased caecal concentrations of the BCFAs isovalerate and isobutyrate derived from the microbial fermentation of BCAAs. In addition, these mice developed insulin resistance and leptinaemia ${ }^{116}$.

By contrast, the microbial metabolite indole specifically derived from the AAA L-tryptophan by the microbial enzyme tryptophanase has been shown to decrease gut inflammation and prevent gut barrier dysfunction $^{117,118}$. In a mouse experiment, acute orally administered indole alleviated lipopolysaccharide-induced upregulation of pro-inflammatory cytokines and downregulated key proteins of the NF- $\mathrm{BB}$ pathway in the liver ${ }^{49}$. However, whether this result can be translated into humans and whether it would result in long-term benefits in patients with NAFLD still needs to be determined.

In an intriguing 2018 publication ${ }^{99}$, faecal metagenome analysis was combined with analysis of the hepatic transcriptome and plasma and urine metabolomes in women with morbid obesity but without evidence of T2DM. Here, the status of steatosis was associated with decreased gut microbial gene richness and dysregulation of microbial AAA and BCAA metabolism. Furthermore, by using a rodent model and human hepatocytes, this study identified microbial-derived phenylacetic acid, a product of phenylalanine catabolism, as a contributor to steatosis progression. Phenylacetic acid might increase hepatic lipid accumulation via a synergetic increase in BCAA utilization in the tricarboxylic acid cycle. These observations again identified proteolytic fermentation products as being important contributors to the development of hepatic steatosis. However, it should be noted that this study also identified other dysbiosis-associated factors, including lipopolysaccharide and trimethylamine $\mathrm{N}$-oxide, as being important contributors to hepatic steatosis. This finding further emphasizes the idea that multifactorial processes related to the microbiome are involved in the progression of metabolic diseases.

Human data in NAFLD. Evidence for a role of the gut microbiome in NAFLD development in humans is mainly derived from cross-sectional observations. On the phylum level, some studies have demonstrated a relationship between an increased Bacteroidetes to Firmicutes ratio and NAFLD progression, but data are not consistent $^{105,119,120}$. On the family level, however, data are more consistent. A number of studies indicated a decrease in butyrate-producing Ruminococcaceae in patients 
with NAFLD ${ }^{105,119,121}$. However, whether NAFLD causes dysbiosis or whether the reverse happens is not yet clear.

Furthermore, direct evidence for a role of the gut microbiome in NAFLD is derived from studies using probiotics and prebiotics ${ }^{122,123}$. Fructooligosaccharide supplementation to the diet of patients with NASH was associated with decreased serum levels of alanine transaminase, aspartate transaminase and insulin after 8 weeks $^{122}$. Furthermore, in patients diagnosed with NAFLD, oral intake of combinations of the acetogenic Bifidobacterium longum and fructooligosaccharide was associated with reductions in steatosis, NASH activity index, serum levels of TNF and serum levels of endotoxin after 24 weeks ${ }^{123}$.

Overall, evidence for a causal role of microbial metabolites in NASH in humans is limited because the gold-standard method for assessing and diagnosing NASH is still a liver biopsy, which means that only small numbers of patients are included in clinical trials using this invasive protocol. Improved noninvasive techniques will help enable well-powered studies to be performed to elucidate the role of the gut microbiome in NAFLD.

\section{Microbial metabolites in T2DM}

In individuals with obesity, T2DM can develop following a progressive rise in insulin resistance and a progressive relative deficiency in insulin secretion. As described above, adipose tissue dysfunction, characterized by

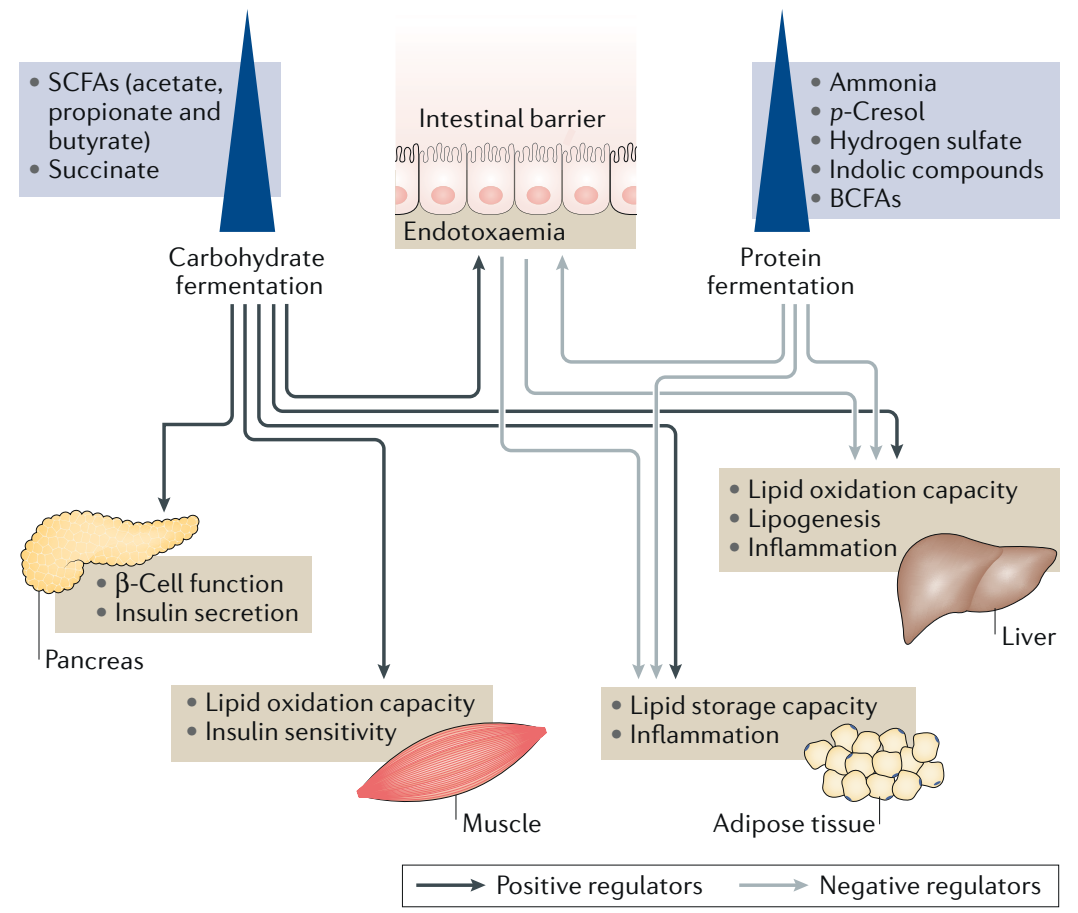

Fig. 3 | The relationship of metabolites derived from protein and carbohydrate fermentation and interorgan crosstalk with insulin resistance and type 2 diabetes mellitus. Carbohydrates and proteins are the major fuels for gut microbial fermentation in the colon. Carbohydrate fermentation mainly occurs in the proximal colon and resulted in the production of gases, short-chain fatty acids (SCFAs) and succinate. The SCFAs acetate, propionate and butyrate as well as succinate are important players in interorgan crosstalk by regulating gut integrity and improving liver and peripheral tissue function and metabolism. Protein fermentation mainly occurs in the distal colon and yields a more diverse range of metabolites, several of which are associated with detrimental effects on gut and metabolic health. BCFAs, branched-chain fatty acids. reduced lipid storage capacity as well as low-grade inflammation and an increase in ectopic fat depots, is specifically associated with insulin resistance ${ }^{124}$. The aforementioned mechanism by which proteolytic and saccharolytic fermentation-derived metabolites affect gut barrier function and the gut immune system in relation to liver metabolism and fat accumulation is not only of importance for NAFLD progression but also can contribute to the development of insulin resistance and T2DM in individuals with obesity ${ }^{14,16,102}$. In this section, we focus on gut microbial products derived from carbohydrate and protein fermentation that affect adipose tissue, skeletal muscle and $\beta$-cell function and metabolism. A schematic view on the role of gut microbial metabolites in T2DM is provided (FIG. 3).

SCFAs affect adipose tissue metabolism. SCFAs have been shown to affect adipose tissue metabolism. In particular, acetate, the systemically most abundant SCFA, might inhibit basal and $\beta$-adrenergic receptor-mediated intracellular lipolysis in adipocytes. The antilipolytic effect of acetate might be caused by decreased phosphorylation of hormone-sensitive lipase in a GPR-dependent manner, as recently demonstrated in $3 \mathrm{~T} 3-\mathrm{L} 1$ rodent cell lines ${ }^{125}$ and in a human adipocyte model ${ }^{126}$. Combined data derived from rodents and humans demonstrated that a partial inhibition of intracellular lipolysis has beneficial effects on insulin sensitivity without affecting adipose tissue mass in the longer term ${ }^{127}$. Nevertheless, the obese insulin-resistant state is characterized by both elevated basal lipolysis and blunted $\beta$-adrenergic receptor-mediated lipolysis. This finding raises the question of whether using SCFAs to further decrease $\beta$-adrenergic receptor-mediated lipolysis in individuals with obesity would have a positive effect on metabolic health. In line with the in vitro findings, several in vivo studies have also shown that acetate can inhibit whole-body lipolysis in humans ${ }^{22}$. For instance, rectal administrations of SCFA mixtures high in acetate decreased circulating concentrations of glycerol ${ }^{30}$. Furthermore, acute intravenously administered acetate decreased plasma levels of free fatty acid in healthy individuals and those who were hyperinsulinaemic and obese ${ }^{128}$.

In addition to its effects on lipolysis, acetate might also increase adipogenesis. Treatment of 3T3-L1 preadipocytes with acetate $(0.1 \mu \mathrm{mol} / \mathrm{l})$ enhanced mRNA expression of genes involved in adipogenic differentiation ${ }^{22}$.

The obese insulin-resistant state is characterized by chronic low-grade inflammation, mainly related to adipose tissue inflammation ${ }^{9}$. Co-incubation of murine 3T3-L1 adipocytes with RAW264.7 macrophages and butyrate for $24 \mathrm{~h}$ reduced TNF, monocyte chemoattractant protein 1 and IL- 6 concentrations ${ }^{129}$. Furthermore, incubation of human adipose tissue explants with propionate attenuated secretion of the pro-inflammatory cytokines IL-4, IL-10, TNF and several chemokines ${ }^{130}$.

In conclusion, evidence exists that SCFAs, in particular acetate, can inhibit lipolysis and increase adipogenesis, thereby improving the lipid storage 
capacity of adipose tissue, preventing lipid overflow and ectopic fat storage. Notably, in view of the blunted catecholamine-induced ( $\beta$-adrenergic receptor-mediated) lipolysis in obesity, further inhibition of lipolysis might not be beneficial under all metabolic conditions, indicating the need for long-term human studies of SCFAs in individuals who are obese, with ectopic fat storage and insulin sensitivity as outcomes. Additionally, SCFAs might attenuate adipose tissue inflammation, thereby preventing low-grade inflammation in the obese insulin-resistant phenotype.

SCFAs affect lipid oxidation capacity in skeletal muscle. As described above, SCFA-induced improvements in adipose tissue metabolism might lead to decreased lipid overflow and intramuscular lipid accumulation and attenuated supply of pro-inflammatory cytokines to the skeletal muscle, preventing insulin resistance. In addition, SCFAs might directly improve skeletal muscle functioning by increasing the skeletal muscle lipid oxidative capacity, as demonstrated in rodents ${ }^{78,131}$. Supplementation with sodium butyrate for 16 weeks to mice fed a high-fat diet increased the proportion of type 1 oxidative muscle fibres and expression of PPAR $\delta$ via PGC1 $\alpha$ activation, resulting in enhanced mitochondrial lipid oxidation ${ }^{78}$. In obese rats, 6 months of acetate treatment improved expression of genes involved in lipid oxidation including increased AMPK activity in skeletal muscle ${ }^{131}$. Thus far, no human data are available demonstrating effects of SCFAs on skeletal muscle lipid oxidation and no data are available on SCFA-induced AMPK and/or PGC1 $\alpha$ activity-related pathways in human skeletal muscle cells.

SCFAs affect $\beta$-cell function and insulin secretion. T2DM is characterized by a decreased capacity to produce the amounts of insulin required to maintain normoglycaemia in the face of insulin resistance. The ability to secrete insulin depends on the functioning and mass of pancreatic $\beta$-cells. Pancreatic $\beta$-cells express the SCFA receptors GPR41 and GPR43 in mice and humans ${ }^{132,133}$. Receptor-knockout experiments in obese and insulin-resistant mice have demonstrated that SCFAs have the capacity to increase glucose-stimulated insulin secretion via GPR43 (REF. ${ }^{132}$ ). In line with this finding, depletion of GPR43 in mice with diet-induced obesity was associated with deteriorated $\beta$-cell function and increased $\beta$-cell mass ${ }^{133}$. Furthermore, SCFA-GPR41 signalling seems to be of particular importance in controlling pancreatic $\beta$-cell insulin secretion in fed and fasting states, as GPR41 knockout or overexpression in mice resulted in impairments in glucose control without effects on insulin sensitivity ${ }^{134}$. Using a propionate inulin ester, a human in vivo study demonstrated that propionate has beneficial effects on $\beta$-cell function and insulin secretion, independent of GLP1 (REF. ${ }^{135}$ ). An in vitro follow-up experiment using human islets demonstrated that propionate potentiated glucose-stimulated insulin release and maintained $\beta$-cell mass through inhibition of apoptosis ${ }^{135}$. Thus, increasing evidence indicates that the SCFA-GPR axis is of major relevance for the control of insulin secretion and $\beta$-cell functioning.
Succinate in insulin resistance. As mentioned earlier, succinate resulted in the activation of IGN in mice fed a high-fat and high-sucrose diet. Microbial-derived succinate not only prevented the obese phenotype but also improved glucose tolerance and insulin sensitivity in wild-type mice. These effects were abolished in mice deficient in IGN (I-G6pc $c^{-1-}$ mice $)^{27}$. By contrast, succinate might act as a metabolite in innate immune signalling. In lipopolysaccharide-activated macrophages, succinate stabilized the transcription factor hypoxia-inducible factor $1 \alpha$, which increased IL- $1 \beta$ production and exacerbated inflammation; therefore, succinate might contribute to progression of insulin resistance ${ }^{136}$. However, whether microbial-derived succinate is of relevance in these processes in humans requires further investigation.

Proteolytic metabolites in insulin resistance and T2DM. An interesting study used computational prediction models in mice to investigate dietary intervention strategies that varied in protein content ${ }^{137}$. The data demonstrated that decreased protein intake and the resulting microbial nitrogen competition promoted a microbial composition associated with improved intestinal function and metabolic health ${ }^{137}$.

Hydrogen sulfide is a major product of protein fermentation. Studies in animal models suggest that excess hydrogen sulfide negatively affects pancreatic islet function, thereby contributing to the development of $\mathrm{T}_{2} \mathrm{DM}^{138,139}$. In addition, hydrogen sulfide stimulates gluconeogenesis and glycogenolysis and decreases glucose utilization and glycogen storage in rodent hepatocyte models, indicating impairments in glucose homeostasis ${ }^{140}$. On the other hand, human data showed that patients with T2DM have reduced plasma concentrations of hydrogen sulfide compared with healthy individuals ${ }^{141}$. In addition, a mouse study demonstrated that hydrogen sulfide has a protective role in pancreatic $\beta$-cells and prevents the onset of T2DM ${ }^{50}$.

Another metabolite specifically originating from proteolytic fermentation by gut microorganisms is $p$-cresol, which is converted to $p$-cresyl sulfate by the host. In mice, administration of $p$-cresyl sulfate for 4 weeks increased ectopic fat accumulation in liver and muscle and triggered peripheral insulin resistance ${ }^{142}$. Interestingly, a study combining cross-sectional analyses of insulin sensitivity, the fasting serum metabolome and the gut microbiome showed that individuals with insulin resistance and T2DM had increased serum levels of the gut microbial-associated phenolic compounds hydrocinnamic acid and indole-3-lactic acid as well as BCAAs ${ }^{51}$. Furthermore, the data demonstrated that the gut microbiome from insulin-resistant individuals had increased BCAA biosynthesis potential (largely driven by Prevotella copri and Bacteroides vulgatus) but a reduced potential for BCAA uptake and catabolism ${ }^{51}$. These data suggest that the microbiome contributes to increased peripheral BCAA concentrations and insulin resistance.

Overall, hydrogen sulfide, $p$-cresol, phenolic compounds and BCAAs derived from proteolytic fermentation seem to be involved in the development of insulin resistance. However, no human intervention studies 
have yet measured the contribution of different protein sources and microbial proteolytic metabolites on host insulin resistance. An interesting approach to perform such a study would be via the use of isotopically labelled complex (indigestible) peptides or proteins.

Human data in insulin resistance and T2DM. Long-term evidence for a direct link between microbial metabolites and insulin resistance is still limited. However, two intriguing dietary intervention studies provide strong indications that SCFAs could be the major driver of the beneficial relationship between fermentable carbohydrate intake and insulin sensitivity ${ }^{17,31}$.

Daily supplementation with $30 \mathrm{~g}$ resistant starch for 4 weeks improved peripheral insulin sensitivity in healthy individuals, which was also associated with increased systemic levels of acetate and propionate and an increase in acetate uptake by adipose tissue and skeletal muscle ${ }^{31}$.

A study published in 2018 showed that a diet rich in complex fibre mixtures for 12 weeks decreased $\mathrm{HbA}_{1 \mathrm{c}}$ levels, fasting blood levels of glucose and glucose tolerance by $\sim 20 \%$ in individuals with $\mathrm{T}_{2} \mathrm{DM}^{17}$. A metagenomics analysis of the faecal microbiota revealed that microbial pathways for acetate and butyrate production were significantly increased in patients following the diet rich in complex fibre mixtures. A trend towards increased faecal acetate and butyrate concentrations in patients following the diet was also seen, and this trend coincided with increased circulating levels of PYY in fasting conditions and GLP1 in postprandial conditions. Interestingly, when responder analyses of the genomes of the faecal bacteria were performed, the positive responders (bacterial strains that were significantly increased after the high-fibre diet) showed an increased genetic microbial capacity to ferment fibres and to produce SCFAs, in particular acetate and butyrate. By contrast, the genome of negative responders (bacterial strains that were decreased after the intervention) harbours genes that are involved in the production of proteolytic metabolites such as indoles and hydrogen sulfide ${ }^{17}$. This finding is in contrast to observations in animals, which have suggested beneficial effects of indolic compounds on gut integrity, liver function and glucose homeostasis ${ }^{49,50}$. This discrepancy should be further investigated in human clinical intervention trials.

In addition, in a pilot study published in 2018 , healthy lean men as well as men with the metabolic syndrome were treated with $4 \mathrm{~g}$ of sodium butyrate daily for 4 weeks ${ }^{35}$. Interestingly, an improvement in peripheral and hepatic insulin sensitivity (determined via hyperinsulinaemiceuglycaemic clamp) was observed in the lean, but not in the metabolically compromised, participant ${ }^{35}$. These pilot data suggest that SCFA handling and/or signalling is disturbed in individuals with the metabolic syndrome. However, the data should be confirmed in a larger cohort and the underlying mechanisms need to be investigated.

\section{Future perspectives}

Consideration of studies investigating the metabolic consequences of saccharolytic and proteolytic microbial fermentation strongly suggests that an increase in metabolites derived from the fermentation of dietary fibre and a decrease in proteolytic products could be a valuable strategy to prevent and/or alleviate metabolic diseases such as obesity, NAFLD and T2DM. However, data on the metabolic benefits of fermentable fibres are inconsistent, which might relate to the characteristics of the specific fibres, including their fermentation type, site of fermentation, amount and/or type of metabolites produced $^{10,143}$. Previous human intervention studies have mainly focused on one specific type of fibre - fermentable oligosaccharides - to alter microbiota composition and elicit metabolic effects. The handful of longer-term human intervention studies using these fermentable fibres alone, however, showed only minor effects ${ }^{144-146}$ or no effects ${ }^{147}$ on circulating SCFA concentrations and insulin sensitivity (indices). Of note, a 2017 study in healthy young individuals showed that fermentable oligosaccharides increased the abundance of bifidobacteria, but decreased the abundance of several other SCFA-producing bacteria, and even resulted in adverse effects on glucose homeostasis ${ }^{148}$. This study suggests two main points: first, that it is important to keep the diversity and abundance of SCFA-producing bacteria high, instead of stimulating only one bacteria genus (even if these bacteria are considered to be beneficial for gut and metabolic health); and second, that the colonic fermentation site of fibres is of importance. In this study, fibre fermentation occurred in the proximal colon owing to the fairly low molecular mass of the products. Interestingly, acute human studies demonstrated that infusions of SCFAs in the distal, but not proximal, colon modulated whole-body substrate metabolism, with an increased lipid oxidation and PYY concentration, and attenuated lipolysis ${ }^{30,34}$.

The study findings do not imply that saccharolytic fermentation in the more proximal colon is not relevant. As shown in animal models, microbial SCFA and succinate production (primarily occurring in the caecum, which equals the proximal colon in humans) is of immense relevance for gut homeostasis and the innate immune system and might be important in the prevention and treatment of NAFLD. However, performing intervention studies would be of interest, focusing on providing both the proximal and distal colonic microbiota its preferred energy source - that is, fermentable dietary fibres. This approach might increase the proliferation of SCFA-producing bacteria and saccharolytic metabolites and inhibit the production of potential harmful metabolites derived from protein fermentation in the distal colon. An innovative fibre mixture to target and reach the distal colonic microbiota might be a combination of readily fermentable oligomers and more complex fermentable fibres with a high degree of polymerization and side chains. The oligomers are the preferred microbial nutritional source and will satisfy the microbiota in the proximal colon, whereas the complex fibres might reach the distal colon. Furthermore, combining such a fibre mixture with SCFA-producing bacteria (a symbiotic intervention) might be another strategy to inhibit products derived from proteolytic fermentation and overgrowth of proteolytic microorganisms.

As the microbiome-host signalling axis might be more resistant to such a fibre (symbiotic) intervention, 
in particular in the obese insulin-resistant phenotype $\mathrm{e}^{35,128,149}$, the approach should be tested for a long time period of $\geq 3$ months ${ }^{17}$. Controlling for factors that could shape the microbial composition and might directly affect host substrate and energy metabolism would also be very important. Such factors include diet and physical activity, which should be assessed or possibly even standardized during the intervention period. In addition, medications, changes in gut transit time, stress, illness, sleep quality and emotional well-being should be (if possible) avoided or at least assessed. Combining in vivo gold-standard clinical techniques such as hyperinsulinaemic-euglycaemic clamp with advanced metabolomics, metatranscriptomics and metagenomics approaches will help to further determine the relationship between the microbiome and host metabolic health.

\section{Conclusions}

An overwhelming amount of animal data provides strong evidence of a beneficial role of the primary saccharolytic-derived microbial fermentation products - the SCFAs acetate, butyrate and propionate - in the prevention of obesity, NAFLD, insulin resistance and T2DM. Furthermore, studies in humans have indicated causality for the metabolic health effects of SCFAs. However, the metabolic consequences of other microbial products derived from saccharolytic fermentation, such as succinate and ethanol, are not yet fully understood. Proteolytic fermentation, which mostly occurs in the distal colon, produces molecules such as BCFAs, phenolic and indolic compounds, ammonia and hydrogen sulfide. These products are far less studied in terms of their metabolic consequences. The limited amount of animal and human data on these metabolites suggests a more detrimental role of these products on gut integrity and metabolic health. Of note, animal data also suggest a beneficial role of proteolytic metabolites such as indole on gut barrier and liver function. A 'microbial substrate switch', increasing dietary fibre availability and SCFA formation in the distal colon and thereby decreasing detrimental proteolytic products, might provide a novel dietary strategy for preventing and/or treating metabolic diseases.

Published online 22 January 2019
1. World Health Organization. Obesity and overweight WHO http://www.who.int/mediacentre/factsheets/ fs311/en/ (2018)

2. Younossi, Z. M. et al. Global epidemiology of nonalcoholic fatty liver disease-meta-analytic assessment of prevalence, incidence, and outcomes. Hepatology 64, 73-84 (2016).

3. Zheng, Y., Ley, S. \& Hu, F. Global aetiology and epidemiology of type 2 diabetes mellitus and its complications. Nat. Rev. Endocrinol. 14, 88-98 (2017).

4. Seuring, T., Archangelidi, O. \& Suhrcke, M The economic costs of type 2 diabetes: a global systematic review. Pharmacoeconomics 33, 811-831 (2015).

5. Tremmel, M., Gerdtham, U.-G., Nilsson, P. M. \& Saha, S. Economic burden of obesity: a systematic literature review. Int. J. Environ. Res. Public Health 14, E435 (2017).

6. Corpeleijn, E., Saris, W. \& Blaak, E. Metabolic flexibility in the development of insulin resistance and type 2 diabetes: effects of lifestyle. Obes. Rev. 10 178-193 (2009)

7. Shulman, G. I. Ectopic fat in insulin resistance, dyslipidemia, and cardiometabolic disease. N. Engl. J. Med. 371, 1131-1141 (2014).

8. Reilly, S. M. \& Saltiel, A. R. Adapting to obesity with adipose tissue inflammation. Nat. Rev. Endocrinol. 13 633-343 (2017)

9. Stinkens, R., Goossens, G. H., Jocken, J. W. \& Blaak, E. E. Targeting fatty acid metabolism to improve glucose metabolism. Obes. Rev. 16, 715-757 (2015).

10. Bäckhed, F. et al. The gut microbiota as an environmental factor that regulates fat storage. Proc Natl Acad. Sci. USA 101, 15718-15723 (2004).

11. Ley, R. E., Turnbaugh, P. J., Klein, S. \& Gordon, J. I. Microbial ecology: human gut microbes associated with obesity. Nature 444, 1022-1023 (2006).

12. Le Chatelier, E. et al. Richness of human gut microbiome correlates with metabolic markers. Nature 500, 541-546 (2013).

13. Dao, M. C. et al. Akkermansia muciniphila and improved metabolic health during a dietary intervention in obesity: relationship with gut microbiome richness and ecology. Gut 65, 426-436 (2016).

14. Cani, P. D. et al. Metabolic endotoxemia initiates obesity and insulin resistance. Diabetes 56 1761-1772 (2007)

15. Cani, P. D. et al. Changes in gut microbiota control metabolic endotoxemia-induced inflammation in high-fat diet-induced obesity and diabetes in mice. Diabetes 57, 1470-1478 (2008)

16. Henao-Mejia, J. et al. Inflammasome-mediated dysbiosis regulates progression of NAFLD and obesity. Nature 482, 179-185 (2012).
17. Zhao, L. et al. Gut bacteria selectively promoted by dietary fibers alleviate type 2 diabetes. Science 359 , 1151-1156 (2018)

18. Kovatcheva-Datchary, P. et al. Dietary fiber-induced improvement in glucose metabolism is associated with increased abundance of Prevotella. Cell Metab. 22, 971-982 (2015)

19. De Vadder, F. et al. Microbiota-generated metabolites promote metabolic benefits via gut-brain neural circuits. Cell 156, 84-96 (2014).

20. Menni, C. et al. Gut microbiome diversity and high-fibre intake are related to lower long-term weight gain. Int. J. Obes. (Lond.) 41, 1099 (2017)

21. Cani, P. D., Joly, E., Horsmans, Y. \& Delzenne, N. M Oligofructose promotes satiety in healthy human: a pilot study. Eur. J. Clin. Nutr. 60, 567-572 (2006).

22. Canfora, E. E., Jocken, J. W. \& Blaak, E. E. Short-chain fatty acids in control of body weight and insulin sensitivity. Nat. Rev. Endocrinol. 11, 577-591 (2015).

23. Koh, A., De Vadder, F., Kovatcheva-Datchary, P. $\&$ Bäckhed, F. From dietary fiber to host physiology: short-chain fatty acids as key bacterial metabolites. Cell 165, 1332-1345 (2016).

24. den Besten, G. et al. Short-chain fatty acids protect against high-fat diet-induced obesity via a PPAR $\gamma$-dependent switch from lipogenesis to fat oxidation. Diabetes 64, 2398-2408 (2015)

25. Frost, G. et al. The short-chain fatty acid acetate reduces appetite via a central homeostatic mechanism. Nat. Commun. 5, 3611 (2014)

26. Lu, Y. et al. Short chain fatty acids prevent high-fat-diet-induced obesity in mice by regulating $G$ protein-coupled receptors and gut microbiota. Sci. Rep. 6, 37589 (2016)

27. De Vadder, F. et al. Microbiota-produced succinate improves glucose homeostasis via intestinal gluconeogenesis. Cell Metab. 24, 151-157 (2016).

28. Mollica, M. P. et al. Butyrate regulates liver mitochondrial function, efficiency, and dynamics in insulin-resistant obese mice. Diabetes 66, 1405-1418 (2017).

29. Li, Z. et al. Butyrate reduces appetite and activates brown adipose tissue via the gut-brain neural circuit. Gut 67, 1269-1279 (2017).

30. Canfora, E. E. et al. Colonic infusions of short-chain fatty acid mixtures promote energy metabolism in overweight/obese men: a randomized crossover trial. Sci. Rep. 7, 2360 (2017).

31. Robertson, M. D., Bickerton, A. S., Dennis, A. L., Vidal, H. \& Frayn, K. N. Insulin-sensitizing effects of dietary resistant starch and effects on skeletal muscle and adipose tissue metabolism. Am. J. Clin. Nutr. 82 , 559-567 (2005)

32. Chambers, E. S. et al. Effects of targeted delivery of propionate to the human colon on appetite regulation, body weight maintenance and adiposity in overweight adults. Gut 64, 1744-1754 (2015).

33. Chambers, E. S. et al. Acute oral sodium propionate supplementation raises resting energy expenditure and lipid oxidation in fasted humans. Diabetes Obes. Metab. 20, 1034-1039 (2018).

34. van der Beek, C. M. et al. Distal, not proximal, colonic acetate infusions promote fat oxidation and improve metabolic markers in overweight/obese men. Clin. Sci. 130, 2073-2082 (2016).

35. Bouter, K et al. Differential metabolic effects of oral butyrate treatment in lean versus metabolic syndrome subjects. Clin. Trans/ Gastroenterol. 9, 155 (2018).

36. Windey, K., De Preter, V. \& Verbeke, K. Relevance of protein fermentation to gut health. Mol. Nutr. Food Res. 56, 184-196 (2012)

37. Russell, W. R. et al. High-protein, reduced-carbohydrate weight-loss diets promote metabolite profiles likely to be detrimental to colonic health. Am. J. Clin. Nutr. 93, 1062-1072 (2011).

38. Wahlström, A., Sayin, S. I., Marschall, H.-U. $\&$ Bäckhed, F. Intestinal crosstalk between bile acids and microbiota and its impact on host metabolism. Cell Metab. 24, 41-50 (2016)

39. Sonnenburg J L \& Bäckhed, F Diet-microbiota interactions as moderators of human metabolism Nature 535, 56-64 (2016).

40. Cani, P. D., Osto, M., Geurts, L. \& Everard, A. Involvement of gut microbiota in the development of low-grade inflammation and type 2 diabetes associated with obesity. Gut Microbes 3, 279-288 (2012).

41. Gilbert, J. A. et al. Current understanding of the human microbiome. Nat. Med. 24, 392-400 (2018)

42. Maier, L. et al. Extensive impact of non-antibiotic drugs on human gut bacteria. Nature 555, 623-628 (2018).

43. Schmidt, T. S., Raes, J. \& Bork, P. The human gut microbiome: from association to modulation. Cell 172, 1198-1215 (2018)

44. Liu, R. et al. Gut microbiome and serum metabolome alterations in obesity and after weight-loss intervention. Nat. Med. 23, 859-868 (2017)

45. Qin, J. et al. A human gut microbial gene catalogue established by metagenomic sequencing. Nature $\mathbf{4 6 4}$ 59-65 (2010)

46. Wong, J. M., de Souza, R., Kendall, C. W., Emam, A $\&$ Jenkins, D. J. Colonic health: fermentation and short chain fatty acids. J. Clin. Gastroenterol. 40, 235-243 (2006).

47. Cummings, J., Pomare, E., Branch, W., Naylor, C. $\&$ Macfarlane, G. Short chain fatty acids in human large intestine, portal, hepatic and venous blood. Gut 28, 1221-1227 (1987).

48. Dodd, D. et al. A gut bacterial pathway metabolizes aromatic amino acids into nine circulating metabolites. Nature 551, 648-652 (2017). 
49. Beaumont, M. et al. The gut microbiota metabolite indole alleviates liver inflammation in mice. FASEB J. 32, 6681-6693 (2018)

50. Okamoto, M. et al. Endogenous hydrogen sulfide protects pancreatic beta-cells from a high-fat diet-induced glucotoxicity and prevents the development of type 2 diabetes. Biochem. Biophys. Res. Commun. 442, 227-233 (2013).

51. Pedersen, H. K. et al. Human gut microbes impact host serum metabolome and insulin sensitivity. Nature 535, 376-381 (2016).

52. Venema, K. Microbial metabolites produced by the colonic microbiota as drivers for immunomodulation in the host. FASEB J. 27 (Suppl. 1), 643.12-643.13 (2013).

53. Pylkas, A. M., Juneja, L. R. \& Slavin, J. L. Comparison of different fibers for in vitro production of short chain fatty acids by intestinal microflora. J. Med. Food $\mathbf{8}$, 113-116 (2005)

54. El Oufir, L. et al. Relationships between transit time in man and in vitro fermentation of dietary fiber by fecal bacteria. Eur. J. Clin. Nutr. 54, 603-609 (2000).

55. Serena, C. et al. Elevated circulating levels of succinate in human obesity are linked to specific gut microbiota. ISME J. 12, 1642-1657 (2018).

56. Bloemen, J. G. et al. Short chain fatty acids exchange: is the cirrhotic, dysfunctional liver still able to clear them? Clin. Nutr. 29, 365-369 (2010).

57. Bloemen, J. G. et al. Short chain fatty acids exchange across the gut and liver in humans measured at surgery. Clin. Nutr. 28, 657-661 (2009).

58. Boets, E. et al. Systemic availability and metabolism of colonic-derived short-chain fatty acids in healthy subjects: a stable isotope study. J. Physiol. 595, 541-555 (2017)

59. Neis, E. P. et al. Distal versus proximal intestinal short-chain fatty acid release in man. Gut https://doi. org/10.1136/gutjnl-2018-316161 (2018).

60. Swanson, K. S. et al. Fructooligosaccharides and Lactobacillus acidophilus modify bowel function and protein catabolites excreted by healthy humans. J. Nutr. 132, 3042-3050 (2002)

61. Pieper, R. et al. Fermentable fiber ameliorates fermentable protein-induced changes in microbial ecology, but not the mucosal response, in the colon of piglets. J. Nutr. 142, 661-667 (2012).

62. Geypens, B. A. et al. Influence of dietary protein supplements on the formation of bacterial metabolites in the colon. Gut 41, 70-76 (1997)

63. Macfarlane, G., Gibson, G. \& Cummings, J. Comparison of fermentation reactions in different regions of the human colon. J. Appl. Bacteriol. 72 , 57-64 (1992)

64. Tolhurst, G. et al. Short-chain fatty acids stimulate glucagon-like peptide-1 secretion via the G-proteincoupled receptor FFAR2. Diabetes 61, 364-371 (2012).

65. Psichas, A. et al. The short chain fatty acid propionate stimulates GLP-1 and PYY secretion via free fatty acid receptor 2 in rodents. Int. J. Obes. (Lond.) 39 424-429 (2015)

66. Reimer, R. A. et al. A human cellular model for studying the regulation of glucagon-like peptide- 1 secretion. Endocrinology 142, 4522-4528 (2001).

67. Larraufie, P. et al. SCFAs strongly stimulate PYY production in human enteroendocrine cells. Sci. Rep. 8, 74 (2018)

68. Zhou, J. et al. Dietary resistant starch upregulates total GLP-1 and PYY in a sustained day-long manner through fermentation in rodents. Am. J. Physiol. Endocrinol. Metab. 295, E1160-E1166 (2008).

69. Forbes, S. et al. Selective FFA2 agonism appears to act via intestinal PYY to reduce transit and food intake but does not improve glucose tolerance in mouse models. Diabetes 64, 3763-3771 (2015).

70. Xiong, Y. et al. Short-chain fatty acids stimulate leptin production in adipocytes through the $G$ protein-coupled receptor GPR41. Proc. Natl Acad. Sci. USA 101, 1045-1050 (2004)

71. Soliman, M. et al. Inverse regulation of leptin mRNA expression by short-and long-chain fatty acids in cultured bovine adipocytes. Domest. Anim. Endocrinol. 33, 400-409 (2007)

72. Al-Lahham, S. H. et al. Regulation of adipokine production in human adipose tissue by propionic acid Eur. J. Clin. Invest. 40, 401-407 (2010).

73. Freeland, K. R. \& Wolever, T. Acute effects of intravenous and rectal acetate on glucagon-like peptide-1, peptide YY, ghrelin, adiponectin and tumour necrosis factor-alpha. Br. J. Nutr. 103, 460-466 (2010)
74. Goswami, C., Iwasaki, Y. \& Yada, T. Short-chain fatty acids suppress food intake by activating vagal afferent neurons. J. Nutr. Biochem. 57, 130-135 (2018)

75. Plamboeck, A. et al. The effect of exogenous GLP-1 on food intake is lost in male truncally vagotomized subjects with pyloroplasty. Am. J. Physiol. Gastrointest. Liver Physiol. 304, G1117-G1127 (2013).

76. Byrne, C. S. et al. Increased colonic propionate reduces anticipatory reward responses in the human striatum to high-energy foods. Am. J. Clin. Nutr. 104, 5-14 (2016)

77. Gulanski, B. I. et al. Increased brain transport and metabolism of acetate in hypoglycemia unawareness. J. Clin. Endocrinol. Metab. 98, 3811-3820 (2013).

78. Gao, Z. et al. Butyrate improves insulin sensitivity and increases energy expenditure in mice. Diabetes 58, 1509-1517 (2009)

79. Sahuri-Arisoylu, M. et al. Reprogramming of hepatic fat accumulation and'browning'of adipose tissue by the short-chain fatty acid acetate. Int. J. Obes. (Lond.) 40, 955-963 (2016)

80. Kondo, T., Kishi, M., Fushimi, T. \& Kaga, T. Acetic acid upregulates the expression of genes for fatty acid oxidation enzymes in liver to suppress body fat accumulation. J. Agr. Food Chem. 57, 5982-5986 (2009).

81. Schwiertz, A. et al. Microbiota and SCFA in lean and overweight healthy subjects. Obesity 18, 190-195 (2010).

82. Teixeira, T. F. et al. Higher level of faecal SCFA in women correlates with metabolic syndrome risk factors. Br. J. Nutr. 109, 914-919 (2013).

83. Turnbaugh, P. J. et al. An obesity-associated gut microbiome with increased capacity for energy harvest. Nature 444, 1027-1031 (2006).

84. den Besten, G. et al. The short-chain fatty acid uptake fluxes by mice on a guar gum supplemented diet associate with amelioration of major biomarkers of the metabolic syndrome. PLOS ONE 9, e 107392 (2014).

85. Pauline, K.-B. \& Rimm, E. B. Whole grain consumption and weight gain: a review of the epidemiological evidence, potential mechanisms and opportunities for future research. Proc. Nutr. Soc. 62, 25-29 (2003).

86. Thompson, S. V., Hannon, B. A., An, R. \& Holscher, H. D. Effects of isolated soluble fiber supplementation on body weight, glycemia, and insulinemia in adults with overweight and obesity: a systematic review and meta-analysis of randomized controlled trials. Am. J. Clin. Nutr. 106, 1514-1528 (2017)

87. Wanders, A. J. et al. Effects of dietary fibre on subjective appetite, energy intake and body weight: a systematic review of randomized controlled trials. Obes. Rev. 12, 724-739 (2011)

88. Parnell, J. A. \& Reimer, R. A. Weight loss during oligofructose supplementation is associated with decreased ghrelin and increased peptide $Y Y$ in overweight and obese adults. Am. J. Clin. Nutr. 89, 1751-1759 (2009).

89. Cani, P. D. et al. Gut microbiota fermentation of prebiotics increases satietogenic and incretin gut peptide production with consequences for appetite sensation and glucose response after a meal. Am. J. Clin. Nutr. 90, 1236-1243 (2009)

90. Daud, N. M. et al. The impact of oligofructose on stimulation of gut hormones, appetite regulation and adiposity. Obesity 22, 1430-1438 (2014).

91. Ridaura, V. K. et al. Gut microbiota from twins discordant for obesity modulate metabolism in mice. Science 341, 1241214 (2013)

92. Newgard, C. B. et al. A branched-chain amino acid-related metabolic signature that differentiates obese and lean humans and contributes to insulin resistance. Cell Metab. 9, 311-326 (2009).

93. Kondo, T., Kishi, M., Fushimi, T., Ugajin, S. \& Kaga, T. Vinegar intake reduces body weight, body fat mass, and serum triglyceride levels in obese Japanese subjects. Biosci. Biotechnol. Biochem. 73, 1837-1843 (2009).

94. Meex, R. C. \& Watt, M. J. Hepatokines: linking nonalcoholic fatty liver disease and insulin resistance. Nat. Rev. Endocrinol. 13, 509-520 (2017).

95. Kelly, C. J. et al. Crosstalk between microbiota-derived short-chain fatty acids and intestinal epithelial HIF augments tissue barrier function. Cell Host Microbe 17, 662-671 (2015).

96. Wang, H.-B., Wang, P.-Y., Wang, X., Wan, Y.-L. \& Liu, Y.-C. Butyrate enhances intestinal epithelial barrier function via up-regulation of tight junction protein Claudin-1 transcription. Dig. Dis. Sci. 57, 3126-3135 (2012).
97. Pussinen, P. J., Havulinna, A. S., Lehto, M., Sundvall, J. \& Salomaa, V. Endotoxemia is associated with an increased risk of incident diabetes. Diabetes Care 34 392-397 (2011)

98. Jayashree, B. et al. Increased circulatory levels of lipopolysaccharide (LPS) and zonulin signify novel biomarkers of proinflammation in patients with type 2 diabetes. Mol. Cell. Biochem. 388, 203-210 (2014).

99. Hoyles, L. et al. Molecular phenomics and metagenomics of hepatic steatosis in non-diabetic obese women. Nat. Med. 24, 1070-1080 (2018).

100. Maslowski, K. M. et al. Regulation of inflammatory responses by gut microbiota and chemoattractant receptor GPR43. Nature 461, 1282-1286 (2009).

101. Macia, L. et al. Metabolite-sensing receptors GPR43 and GPR 109A facilitate dietary fibre-induced gut homeostasis through regulation of the inflammasome. Nat. Commun. 6, 6734 (2015)

102. Tremaroli, V. \& Bäckhed, F. Functional interactions between the gut microbiota and host metabolism. Nature 489, 242-249 (2012)

103. Yamashita, H. et al. Improvement of obesity and glucose tolerance by acetate in type 2 diabetic Otsuka Long-Evans Tokushima Fatty (OLETF) rats. Biosci. Biotechnol. Biochem. 71, 1236-1243 (2007).

104. Sakakibara, S., Yamauchi, T., Oshima, Y., Tsukamoto, Y. $\&$ Kadowaki, T. Acetic acid activates hepatic AMPK and reduces hyperglycemia in diabetic KK-A (y) mice. Biochem. Biophys. Res. Commun. 344, 597-604 (2006).

105. Zhu, L. et al. Characterization of gut microbiomes in nonalcoholic steatohepatitis (NASH) patients: a connection between endogenous alcohol and NASH. Hepatology 57, 601-609 (2013).

106. Nair, S., Cope, K., Terence, R. H. \& Diehl, A. M. Obesity and female gender increase breath ethano concentration: potential implications for the pathogenesis of nonalcoholic steatohepatitis. Am. J. Gastroenterol. 96, 1200-1204 (2001).

107. Zhu, L., Baker, R. D., Zhu, R. \& Baker, S. S. Gut microbiota produce alcohol and contribute to NAFLD. Gut 65, 1232 (2016)

108. Xu, J. et al. Synergistic steatohepatitis by moderate obesity and alcohol in mice despite increased adiponectin and p-AMPK. J. Hepatol. 55, 673-682 (2011).

109. Baker, S. S., Baker, R. D., Liu, W., Nowak, N. J. \& Zhu, L. Role of alcohol metabolism in non-alcoholic steatohepatitis. PLOS ONE 5, e9570 (2010).

110. Rao, R., Seth, A. \& Sheth, P. Recent advances in alcoholic liver disease I. Role of intestinal permeability and endotoxemia in alcoholic liver disease. $A m$. $J$. Physiol. Gastrointest. Liver Physiol. 286, G881-G884 (2004).

111. Shen, Z. et al. Role of SIRT1 in regulation of LPS-or two ethanol metabolites-induced TNF- $\alpha$ production in cultured macrophage cell lines. Am. J. Physiol. Gastrointest. Liver Physiol. 296, G1047-G1053 (2009).

112. Blanco, A. M., Perez-Arago, A., Fernandez-Lizarbe, S. $\&$ Guerri, C. Ethanol mimics ligand-mediated activation and endocytosis of IL-1 RI/TLR4 receptors via lipid rafts caveolae in astroglial cells. J. Neurochem. 106, 625-639 (2008).

113. Round, J. L. \& Mazmanian, S. K. The gut microbiota shapes intestinal immune responses during health and disease. Nat. Rev. Immunol. 9, 313-323 (2009).

114. Yao, C., Muir, J. \& Gibson, P. Insights into colonic protein fermentation, its modulation and potential health implications. Aliment. Pharmacol. Ther. 43 181-196 (2016).

115. Andriamihaja, M. et al. Colon luminal content and epithelial cell morphology are markedly modified in rats fed with a high-protein diet. Am. J. Physiol. Gastrointest. Liver Physiol. 299, G1030-G1037 (2010).

116. Le Roy, T. et al. Intestinal microbiota determines development of non-alcoholic fatty liver disease in mice. Gut 62, 1787-1794 (2013).

117. Bansal, T., Alaniz, R. C. Wood, T. K. \& Jayaraman, A. The bacterial signal indole increases epithelial-cell tight-junction resistance and attenuates indicators of inflammation. Proc. Natl Acad. Sci. USA 107 228-233 (2010)

118. Cervantes-Barragan, L. et al. Lactobacillus reuteri induces gut intraepithelial $\mathrm{CD} 4^{+} \mathrm{CD} 8 \alpha \alpha^{+} \mathrm{T}$ cells. Science 357, 806-810 (2017)

119. Wang, B. et al. Altered fecal microbiota correlates with liver biochemistry in nonobese patients with 
non-alcoholic fatty liver disease. Sci. Rep. 6, 32002 (2016)

120. Mouzaki, M. et al. Intestinal microbiota in patients with nonalcoholic fatty liver disease. Hepatology $\mathbf{5 8}$ 120-127 (2013).

121. Silva, H. E. et al. Nonalcoholic fatty liver disease is associated with dysbiosis independent of body mass index and insulin resistance. Sci. Rep. 8, 1466 (2018).

22. Daubioul, C., Horsmans, Y., Lambert, P., Danse, E. $\&$ Delzenne, N. M. Effects of oligofructose on glucose and lipid metabolism in patients with nonalcoholic steatohepatitis: results of a pilot study. Eur. J. Clin. Nutr. 59, 723-726 (2005).

123. Malaguarnera, $M$. et al. Bifidobacterium longum with fructo-oligosaccharides in patients with non alcoholic steatohepatitis. Dig. Dis. Sci. 57, 545-553 (2012).

124. DeFronzo, R. et al. The effect of insulin on the disposal of intravenous glucose: results from indirect calorimetry and hepatic and femoral venous catheterization. Diabetes 30, 1000-1007 (1981)

125. Aberdein, N., Schweizer, M. \& Ball, D. Sodium acetate decreases phosphorylation of hormone sensitive lipase in isoproterenol-stimulated 3T3-L1 mature adipocytes. Adipocyte 3, 121-125 (2014)

126. Jocken, J. W. et al. Short-chain fatty acids differentially affect intracellular lipolysis in a human white adipocyte model. Front. Endocrinol (Lausanne) 8, 372 (2017).

127. Girousse, A. et al. Partial inhibition of adipose tissue lipolysis improves glucose metabolism and insulin sensitivity without alteration of fat mass. PLOS Biol. 11, e1001485 (2013)

128. Fernandes, J., Vogt, J. \& Wolever, T. M. Intravenous acetate elicits a greater free fatty acid rebound in normal than hyperinsulinaemic humans. Eur. J. Clin. Nutr. 66, 1029-1034 (2012).

129. Ohira, H. et al. Butyrate attenuates inflammation and lipolysis generated by the interaction of adipocytes and macrophages. J. Atheroscler. Thromb. 20, 425-442 (2013)

130. Al-Lahham, S.a. et al. Propionic acid affects immune status and metabolism in adipose tissue from overweight subjects. Eur. J. Clin. Invest. 42, 357-364 (2012).

131. Yamashita, H. et al. Effects of acetate on lipid metabolism in muscles and adipose tissues of type 2 diabetic Otsuka Long-Evans Tokushima Fatty (OLETF) rats. Biosci. Biotechnol. Biochem. 73, 570-576 (2009).

132. Priyadarshini, M. et al. An acetate-specific GPCR, FFAR2, regulates insulin secretion. Mol. Endocrinol. 29, 1055-1066 (2015)
133. McNelis, J. C. et al. GPR43 potentiates $\beta$-cell function in obesity. Diabetes 64, 3203-3217 (2015).

134. Veprik, A., Laufer, D., Weiss, S., Rubins, N. \& Walker, M. D. GPR41 modulates insulin secretion and gene expression in pancreatic $\beta$-cells and modifies metabolic homeostasis in fed and fasting states. FASEB J. 30, 3860-3869 (2016).

135. Pingitore, A. et al. The diet-derived short chain fatty acid propionate improves beta-cell function in humans and stimulates insulin secretion from human islets in vitro. Diabetes Obes. Metab. 19, 257-265 (2017).

136. Tannahill, G. et al. Succinate is an inflammatory signal that induces IL-1 $\beta$ through HIF- $1 \alpha$. Nature 496 , 238-242 (2013)

137. Holmes, A. J. et al. Diet-microbiome interactions in health are controlled by intestinal nitrogen source constraints. Cell Metab. 25, 140-151 (2017).

138. Wu, L. et al. Pancreatic islet overproduction of $\mathrm{H} 2 \mathrm{~S}$ and suppressed insulin release in Zucker diabetic rats. Lab. Invest. 89, 59-67 (2009).

139. Yang, G., Yang, W., Wu, L. \& Wang, R. H2S endoplasmic reticulum stress, and apoptosis of insulin-secreting beta cells. J. Biol. Chem. 282 16567-16576 (2007).

140. Zhang, L. et al. Hydrogen sulfide impairs glucose utilization and increases gluconeogenesis in hepatocytes. Endocrinology 154, 114-126 (2013).

141. Jain, S. K. et al. Low levels of hydrogen sulfide in the blood of diabetes patients and streptozotocin-treated rats causes vascular inflammation? Antioxid. Redox Signal. 12, 1333-1338 (2010).

142. Koppe, L. et al. p-Cresyl sulfate promotes insulin resistance associated with CKD. J. Am. Soc. Nephrol. 24, 88-99 (2013).

143. Müller, M., Canfora, E. E. \& Blaak, E. E. Gastrointestinal transit time, glucose homeostasis and metabolic health: modulation by dietary fibers. Nutrients 10, E275 (2018).

144. Dewulf, E. et al. Insight into the prebiotic concept: lessons from an exploratory, double blind intervention study with inulin-type fructans in obese women. Gut 62, 1112-1121 (2013)

145. Vulevic, J., Juric, A., Tzortzis, G. \& Gibson, G. R. A mixture of trans-galactooligosaccharides reduces markers of metabolic syndrome and modulates the fecal microbiota and immune function of overweight adults. J. Nutr. 143, 324-331 (2013).

146. Canfora, E. E. \& Blaak, E. E. The role of polydextrose in body weight control and glucose regulation. Curr. Opin. Clin. Nutr. Metab. Care 18, 395-400 (2015).
147. Canfora, E. E. \& van der Beek, C. M. Supplementation of diet with galacto-oligosaccharides increases bifidobacteria, but not insulin sensitivity, in obese prediabetic individuals. Gastroenterology 153, 87-97 (2017).

148. Liu, F. et al. Fructooligosaccharide (FOS) and galactooligosaccharide (GOS) increase bifidobacterium but reduce butyrate producing bacteria with adverse glycemic metabolism in healthy young population. Sci. Rep. 7, 11789 (2017).

149. Canfora, E. E. \& Blaak, E. E. Acetate: a diet-derived key metabolite in energy metabolism: good or bad in context of obesity and glucose homeostasis? Curr. Opin. Clin. Nutr. Metab. Care 20, 477-483 (2017).

\section{Author contributions}

All authors provided a substantial contribution to the discussion of content; E.E.C. researched data for the article and wrote the article; and R.C.R.M., K.V. and E.E.B. reviewed and edited the manuscript before submission.

Competing interests

The authors declare no competing interests.

Publisher's note

Springer Nature remains neutral with regard to jurisdictional claims in published maps and institutional affiliations.

\section{Review criteria}

PubMed and Google Scholar were searched for relevant topics, using the search terms "SCFA" "acetate" "butyrate", "propionate", "succinate", "dietary fibre", probiotics", "BCFA", "ethanol", “indoles","amines", "sulfate", "choline", "bile acids" and "proteolytic fermentation" in combination with "intestinal concentrations", "blood concentrations", "microbiota", "fermentation", "intestinal homeostasis", "obesity", "NASH", NAFLD", "weight", "satiety", "type 2 diabetes", "insulin sensitivity", "insulin resistance", "glycaemic control", "glucose-lowering mechanisms", "energy metabolism", "inflammation", "treg”, "vagal activity", "cardiovascular disease" and "metabolic control", without publication time constraints. References cited in this article include English-language original research and in some specific case reviews by experts in the field.

Reviewer information

Nature Reviews Endocrinology thanks G. Frost and other anonymous reviewer(s) for their contribution to the peer review of this work. 